\title{
Peptides \\ antimicrobiens \\ naturels cutanés
}

> Une des stratégies majeures mises en jeu par Jens-Michael Schröder, Jürgen Harder la peau pour se protéger des infections est de maintenir une barrière physique cutanée intacte. La désquamation, de même que la sécrétion de mucus, sont à l'origine d'un renouvellement permanent de la surface cutanée, qui s'accompagne d'une élimination des micro-organismes adhérant à cette surface. La découverte récente de peptides antibiotiques épithéliaux suggère qu'il existe, à côté de cette barrière physique, une barrière cutanée «chimique » constituée par des peptides antibiotiques capables de contrôler la croissance bactérienne au niveau des couches cutanées et, éventuellement, de lutter contre les infections. <

\section{Peptides et protéines antimicrobiens cutanés en situation non pathologique}

L'analyse systématique de couche cornée extraite de sujets sains, à la recherche de peptides ou de protéines antimicrobiens (AMP) constitutifs, a permis l'identification du lysozyme, une protéine antimicrobienne exprimée par les kératinocytes [1, 2]. Mais le principal peptide antimicrobien est la RNase 7, une protéine hautement basique dont la structure est similaire à celle des membres de la superfamille de la RNase A, parmi lesquels on retrouve la RNase 2 (EDN, eosinophil-derived neurotoxin), la RNase 3 ( $(\mathrm{CP}$, eosinophil-derived cationic protein) et la RNase 5 (angiogénine) [3].

La RNase 7, dont la teneur dans la couche cornée saine varie de 4 à $8 \mathrm{mg} / \mathrm{g}$, présente un large spectre d'activité antimicrobienne [3]. Elle tue avec une grande efficacité une souche d'Enterococcus faecium résistance à la vancomycine, et ce dès une concentration de $20 \mathrm{nM}$ : d'un point de vue molaire, la RNase 7 est donc la plus puissante et la plus efficace des protéines antimicrobiennes humaines connues aujourd'hui. Elle est exprimée par les kératinocytes de la peau humaine et de divers autres tissus épithéliaux [3], et son expres-

Article reçu le 30 septembre 2005, accepté le 16 novembre 2005. Article traduit par Emmanuelle Chollet. chollet@necker.fr

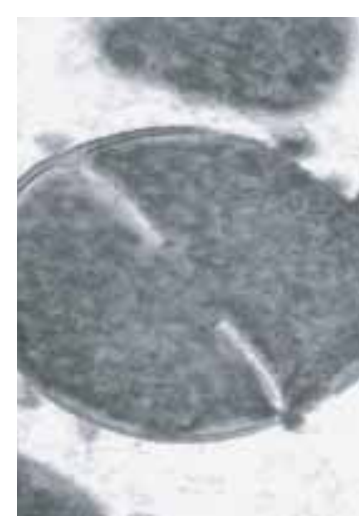

Clinical research unit, Department of Dermatology, University Hospital Schleswig-Holstein, Campus Kiel, Schittenhelmstrasse 7, D-24105 Kiel, Allemagne. jschroeder@

sion peut être induite dermatology.uni-kiel.de

\section{inflammatoires ou par} des bactéries; le mécanisme moléculaire par lequel ces dernières déclenchent la production de RNase 7 par les kératinocytes reste toutefois inconnu.

L'efficacité de la RNase 7 à tuer des entérocoques à des concentrations de l'ordre du nanomolaire suggère qu'elle pourrait jouer un rôle de protection de la peau vis-à-vis des bactéries intestinales à Gram. De fait, il est rare que des bactéries intestinales colonisent les surfaces cutanées; de plus, certaines bactéries y meurent rapidement, tandis que d'autres y survivent [4]. La peau humaine saine semble ainsi capable de produire, de façon constitutive, une arme contre $\varepsilon$. coli, mais pas contre S. aureus (Figure 1) [5] : cette arme est la psoriasine, une protéine de la famille des protéines $S 100$ (S100-A7).

La psoriasine est notamment produite dans les zones où la colonisation bactérienne est abondante (Figure 2), ainsi que dans les glandes sébacées, ce qui suggère qu'elle pourrait être co-sécrétée avec les lipides. L'extraction par l'acétone de zones cutanées riches en lipides aboutit effectivement à l'obtention d'une grande quantité de psoriasine, qui semble donc également stockée dans la couche lipidique de la peau saine. La psoriasine, qui possède une activité antimicrobienne essentiellement dirigée contre $\varepsilon$. coli, avec une $\mathrm{LD}_{50}$ (dose entraînant la destruction de $50 \%$ des souches) proche de $0,5 \mu \mathrm{M}$, est probablement le principal 
composant bactéricide de la peau: le traitement de volontaires sains par un anticorps neutralisant la psoriasine entraîne d'ailleurs une augmentation de la croissance d' $\varepsilon$. coli.

Les glandes sudoripares eccrines produisent quant à elles un peptide antimicrobien, la dermcidine, produite et exprimée de façon constitutive sous la forme d'un précurseur se transformant en peptide actif dans la sueur, après protéolyse [6].

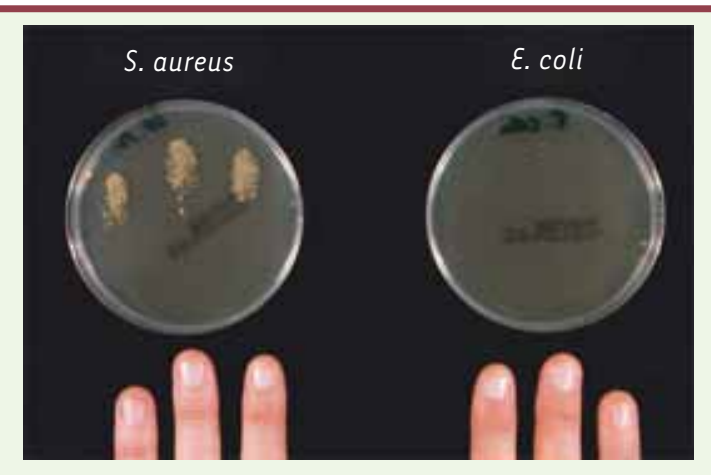

Figure 1. Survie de S. aureus ou $\varepsilon$. coli à la surface de la peau. Les extrémités des doigts, lavés, d'un volontaire sain sont inoculées artificiellement avec une souche de $S$. aureus ou d'E. coli. Après exposition pendant 30 minutes, les extrémités des doigts sont appliquées sur un gel d'agar, et les colonies de $S$. aureus (à gauche) ou $\varepsilon$. coli (à droite) dénombrées (d'après [5]). $\varepsilon$. coli n'est pas transféré, suggérant que la peau humaine produit de façon constitutive une défense chimique capable de tuer les souches d'E. coli.

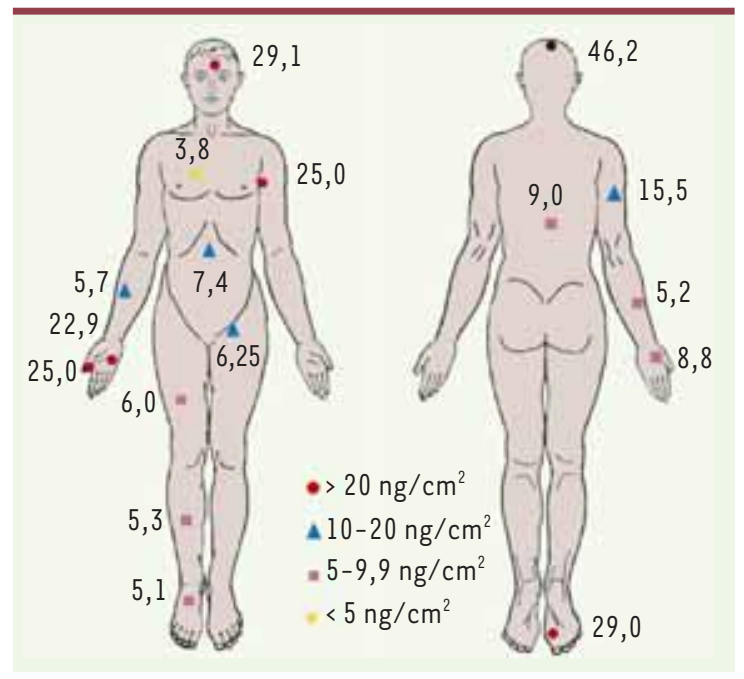

Figure 2. Concentrations locales de psoriasine en différents sites de l'organisme humain sain. Des zones standardisées situées en différents points de l'organisme de volontaires sains ont été lavées avec un tampon de phosphate de $\mathrm{Na}(10 \mathrm{mM}, \mathrm{pH} 7,4)$, et leur concentration en psoriasine déterminée (d'après [5]).

\section{hBD-2, premier peptide antimicrobien épithélial inductible identifié chez l’homme}

La $\beta$-défensine 2 humaine (hBD-2), un peptide de $4 \mathrm{kDa}$ se liant à I'héparine [7], représente l'un des principaux peptides antimicrobiens contenus dans les extraits squameux provenant de lésions psoriasiques (pour revue, voir [8]). Inactive face à $S$. aureus, hBD-2 possède une activité antimicrobienne essentiellement dirigée contre diverses bactéries à $\mathrm{Gram}^{-}$; cette activité est toutefois inhibée par une concentration en $\mathrm{NaCl}$ de 150 mM [9], et donc probablement absente dans le sérum ou sur les surfaces cutanées recouvertes de sueur évaporée. Le peptide hBD-2 est notamment exprimé par certaines cellules différenciées de la peau inflammatoire [10], ce qui suggère qu'elle est induite localement, et qu'un état de différenciation et d'inflammation suffisant est nécessaire à cette induction [11]. Dans les cellules épithéliales, les interleukines (IL) $1 \alpha$ et $1 \beta$, de même qu'une souche vivante de Pseudomonas aeruginosa, sont les inducteurs les plus puissants, compatibles avec ce qui pourrait être observé in vivo, de la production d'hBD-2 [11-14]. Les mécanismes utilisés par les bactéries pour induire la production d'hBD-2 reste toutefois non élucidés; bien que le CD14 et les récepteurs Toll-like (TLR) aient été proposés comme mécanismes importants dans le système de reconnaissance, il existe aujourd'hui une controverse quant à l'implication directe des TLR dans l'induction d'hBD-2 in vivo. En cas d'inflammation, cependant, il est probable que l'induction d'hBD-2 intervienne principalement via la signalisation par l'IL 1 dérivée des phagocytes mononucléaires [13].

Les bactéries peuvent également stimuler directement la production épithéliale d'hBD-2, comme cela a été montré, pour diverses bactéries, dans des cellules épithéliales de la bouche [15, 16]. Dans une étude portant sur des cellules épithéliales issues du tractus respiratoire, seul un isolat clinique de $P$. aeruginosa, de phénotype mucoïde, était capable d'induire la production d'hBD-2 [9]. Ces résultats suggèrent que, hormis les ligands activateurs de TLR, d'autres molécules encore inconnues peuvent induire la production d'hBD-2, probablement même en l'absence d'inflammation.

\section{hBD-3, un peptide antimicrobien inductible au large spectre}

La défensine- $\beta 3$ humaine (hBD-3), un peptide de $5 \mathrm{kDa}$ hautement basique ( $\mathrm{pl} 10,08)$ initialement découvert dans des extraits squameux de lésions psoriasiques [17], présente une activité antimicrobienne à large spectre, puissante et sensible au $\mathrm{NaCl}$, et dirigée contre beaucoup de bactéries à $\mathrm{Gram}^{+}$ou à $\mathrm{Gram}^{-}$et de champignons, certaines de ces souches étant résistantes. Le mécanisme par lequel hBD-3 tue $S$. aureus est, là encore, inconnu. L'analyse ultrastructurale de $S$. aureus soumis à l'action d'hBD-3 révèle des modifications morphologiques similaires à celles observées quand la bactérie est mise en présence de pénicilline (Figure 3) [17].

L'ARN messager de hBD-3 est exprimé dans les épithélium de divers organes, ainsi que dans certains tissus non épithéliaux [17]. Bien que le petide soit une $\beta$-défensine inductible, son expression est régulée 
différemment de celle d'hBD-2. Dans les kératinocytes, hBD-3 est modérément induite par le TNF $\alpha$, et plus puissamment par l'interféron $\gamma$ [18]. L'IGF-I (insulin-like growth factor) et le TGF $\beta$ (transforming growth factor) induisent également la synthèse d'hBD-3, par un mécanisme de transactivation (relargage du TGF $\alpha$ par un processus dépendant d'une métalloprotéase) du récepteur de I'EGF (epidermal growth factor) [13].

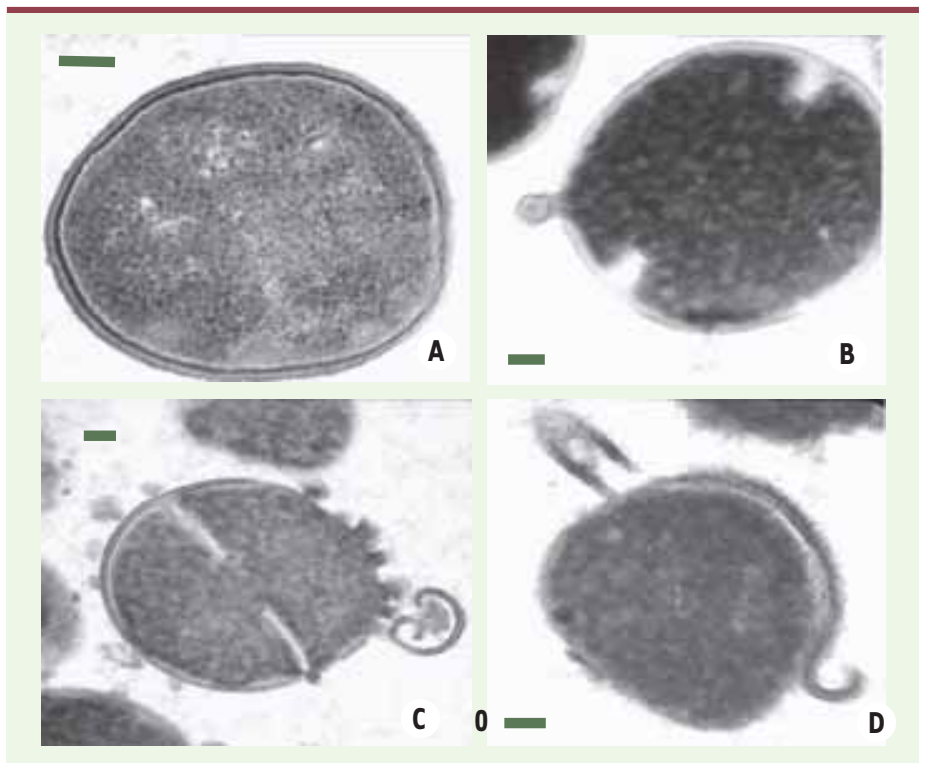

Figure 3. Modifications morphologiques observées sur S. aureus traité par hBD3. Analyse par microscopie électronique à transmission d'une souche de $S$. aureus incubée pendant 2 heures en tampon phosphate $10 \mathrm{mM}$ (A) ou traitée par le peptide synthétique hBD-3 pendant 30 minutes ( $B$ ) ou 2 heures ( $C$ et $D$ ). Des signes de perforation de la paroi cellulaire sont visibles, avec une libération quasi-explosive de la membrane plasmique dès 30 minutes d'incubation ( $B$ ). Au bout de 2 heures ( $C$ et $D$ ), la plupart des cellules subissent une bactériolyse et une désintégration à différents degrés. Ces effets ressemblent à ceux observés lorsqu'une souche de S. aureus est traitée avec de la pénicilline [28], un antibiotique qui interfère avec la réticulation des peptidoglycanes de la paroi cellulaire bactérienne. Les barres correspondent à $0,1 \mu \mathrm{m}$ (d'après [17]).

\section{La cathélicidine humaine hCAP-18/LL-37}

Les cathélicidines sont des molécules contenant un domaine cathelin (cathepsin L inhibitor) à leur extrémité aminoterminale et, à leur extrémité carboxyterminale, un domaine hautement cationique, $\alpha$ hélicoïdal, portant une activité antimicrobienne. Un seul gène codant pour une cathélicidine a pu être retrouvé chez l'homme [19]. Son produit, hCAP-18 (human cathelicidin antimicrobial protein), est une des protéines majeures des granules secondaires des polynucléaires neutrophiles, dans lesquels elle est stockée sous forme d'un précurseur qui subira, dans un second temps, une protéolyse par la protéinase 3 pour donner naissance à son peptide carboxyterminal, le LL-37. Bien que les neutrophiles constituent la source cellulaire majeure d'hCAP-18, cette protéine est également exprimée dans la peau et dans d'autres tissus épithéliaux (pour revue, voir [20]); non exprimée dans la peau saine, hCAP-18 est induite dans les kératinocytes localisés dans les zones inflammatoires au cours de divers troubles cutanés inflammatoires [21], où elle est stockée dans les granules lamellaires cutanés [22]. Cette induction de l'expression d'hCAP-18 par les kératinocytes impliquent l'intervention de I'IGF-I, ainsi que celle du TGF $\alpha$ ou de la 1,25-dihydroxyvitamine D3 [23].

In vitro, hCAP-18 inhibe, à des concentrations de l'ordre du $\mathrm{mM}$, la croissance de diverses bactéries à $\mathrm{Gram}^{-}$ou à Gram ${ }^{+}$Quant à l'implication des cathélicidines in vivo, elle a été démontrée dans un modèle murin: les souris n'exprimant plus la protéine Cramp (cathelicidin-related antimicrobial peptide, homologue murin de LL37) sont plus suceptibles que les souris sauvages aux infections cutanées causées par des streptocoques du groupe A [24].

\section{Rôle des peptides antimicrobiens dans les maladies cutanées}

Dans l'hypothèse où les peptides antimicrobiens naturels sont d'importants effecteurs de la «barrière chimique » mise en place pour protéger la peau des infections comme de l'inflammation, il est logique de spéculer que des déficiences dans ce système de protection pourraient conduire à la survenue d'infections locales récurrentes. Inversement, on peut supposer qu'une surexpression de ces peptides, comme c'est le cas au cours du psoriasis [8], surprotège contre les infections cutanées: de fait, les infections cutanées chez les patients atteints de psoriasis sont rares [25]. À l'opposé, les patients atteints d'eczéma atopique, chez qui une diminution de l'expression d'hBD-2 et -3 et de LL37 est observée dans les lésions aiguës et chroniques, souffrent souvent d'infections causées par $S$. aureus [26, 27]. II est possible que l'augmentation de l'expression des peptides antimicrobiens, normalement attendue dans des lésions inflammatoires atopiques, soit en réalité inhibée par les concentrations élevées de cytokines de type Th2, susceptibles d'inhiber l'induction d'hBD-2 et -3 .

\section{Conclusions et perspectives}

La défense naturelle de la peau semble donc être, chez I'homme sain, en partie assurée par une «barrière chimique », composée de peptides et de protéines antimicrobiens (AMP) exprimés dans l'épiderme, soit de façon constitutive dans les zones les plus superficielles, et 
stockés dans la couche cornée, soit de façon inductible, dans la partie plus profonde, vivante, de l'épiderme (Figure 4).

La découverte que la psoriasine, qui est bactéricide en entraînant une déprivation en zinc, est la principale protéine cutanée antimicrobienne chez l'homme [5] suggère que des déprivations en oligo-éléments essentiels pourraient servir de fondement à de nouvelles stratégies visant à contrôler la croissance microbienne sur les surfaces cutanées (hors zones de désquamation).

L'expression localisée de différents AMP dans la peau saine, dénuée de tout signe visible d'inflammation, conduit également à supposer qu'il existe des situations où la production d'AMP est induite en l'absence de cytokines pro-inflammatoires ou de facteurs de croissance produits durant la cicatrisation, et que des micro-organismes présents sur les surfaces cutanées pourraient faciliter l'induction d'AMP en l'absence de toute inflammation. Des composants de ces bactéries, capables d'initier la production épithéliale d'AMP, pourraient alors être utilisés pour augmenter la résistance cutanée aux infections. $\diamond$ of these AMPs can be induced in vitro in epithelial cells by proinflammatory cytokines or bacteria. In vivo, AMPs are mainly expressed in uppermost and differentiated parts of inflammatory lesions and wounds, but some are also focally expressed in skin in the absence of inflammation, suggesting that apart from inflammatory mediators, also non-inflammatory stimuli of endogenous and/or exogenous origin can stimulate AMP-synthesis. Increased levels of AMPs in psoriatic lesions may explain why psoriasis patients rarely suffer from skin infections. Further, an increased infection rate in atopic dermatitis patients could be the consequence of decreased levels of AMPs in atopic lesions. These observations may indicate an important role of the "chemical skin barrier" in prevention of skin infection and suggest that artificial stimulation of this system, without inflammation, would be beneficial as «immune stimulus ». $\diamond$

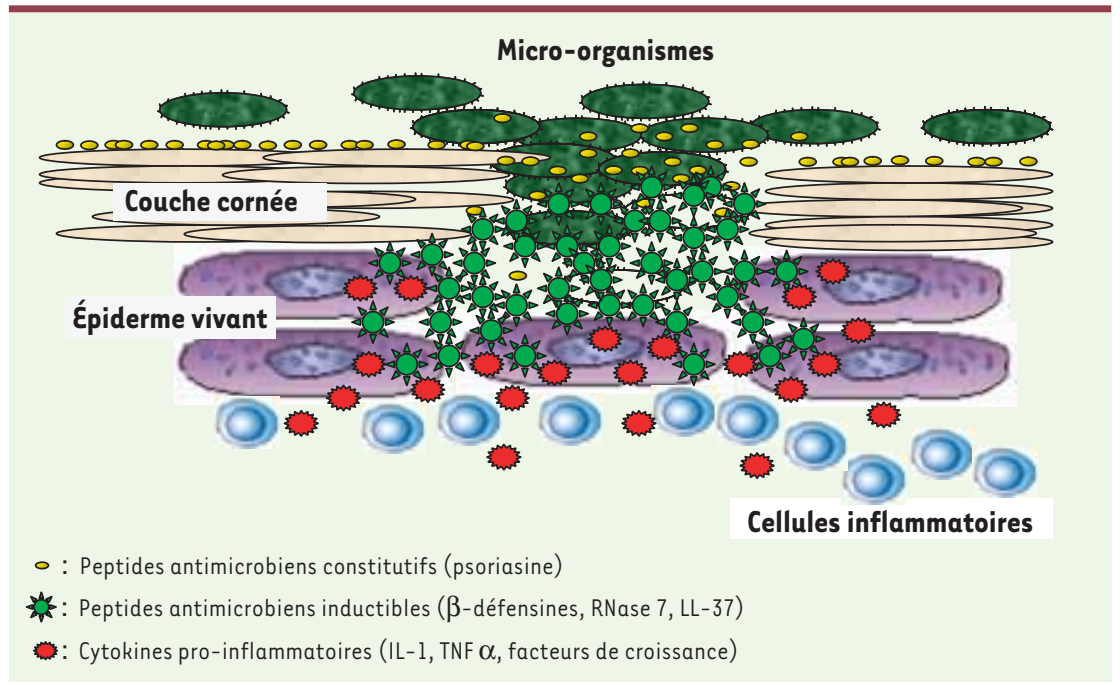

Figure 4. Organisation de la «barrière chimique » cutanée. Les bactéries résidant à la surface de la peau sont prises en charge par des peptides antimicrobiens constitutifs (notamment par la psoriasine). Lorsque la barrière physique est détruite, les bactéries ou leurs dérivés induisent la production de cytokines pro-inflammatoires ou de peptides (ou protéines) antimicrobiens par les kératinocytes (et les leucocytes infiltrés).

\section{SUMMARY}

Innate antimicrobial peptides in the skin

Human skin is always in contact with the environment and is covered with a characteristic microflora, but it is usually not infected. Although desquamation and secretion of mucus lead to a permanent renewal of these body surfaces and simultaneous elimination of microorganisms adhering to these layers, another reason for this natural resistance might be the existence of a "chemical barrier" consisting in constitutively and inducibly produced antimicrobial peptides and proteins (AMPs), which include some ß-defensins, RNase 7, the S100-protein psoriasin and the cathelicidin LL-37. Most

\section{RÉFÉRENCES}

1. Elias PM. Stratum corneum defensive functions: an integrated view. J Invest Dermatol 2005 ; $125: 183-200$.

2. Ogawa H, Miyazaki H, Kimura M. Isolation and characterization of human skin lysozyme. J Invest Dermatol 1971; 57 : 111-6.

3. Harder J, Schröder JM. RNase 7, a novel innate immune defense antimicrobial protein of healthy human skin. J Biol Chem 2002 ; 277 : 46779-84.

4. Casewell MW, Desai N. Survival of multiply-resistant Klebsiella aerogenes and other gram negative bacilli on finger-tips. J Hosp Infect 1983 ; 4 : 350-60.

5. Gläser R, Harder J, Lange $H$, et al. Antimicrobial psoriasin (S100A7) protects human skin from Escherichia coli infection. Nat Immunol 2005; $6: 57-64$.

6. Schittek B, Hipfel R, Sauer B, et al. Dermcidin: a novel human antibiotic peptide secreted by sweat glands. Nat Immunol $2001 ; 2$ : 1133-7.

7. Harder J, Bartels J, Christophers $\varepsilon$, et al. A peptide antibiotic from human skin. Nature 1997; 387 : 861

8. Harder J, Schröder JM. Psoriatic scales: a promising source for the isolation of human skin-derived antimicrobial proteins. J Leukoc Biol 2005 ; $77: 476-86$

9. Harder J, Meyer-Hoffert U, Teran LM, et al. Mucoid Pseudomonas aeruginosa, TNF-alpha, and IL-lbeta, but not IL-6, induce human beta-defensin- 2 in respiratory epithelia. Am J Respir Cell Mol Biol 2000 ; $22: 714-21$

10. Ali RS, Falconer A, Ikram M, et al. Expression of the peptide antibiotics human beta defensin-1 and human beta defensin-2 in normal human skin. J Invest Dermatol $2001 ; 117$ : 106-11.

11. Harder J, Meyer-Hoffert U, Wehkamp K, et al. Differential gene induction of human beta-defensins ( $h B D-1,-2,-3$, and -4 ) in keratinocytes is inhibited by retinoic acid. J Invest Dermatol 2004 ; 123 : 522-9.

12. Becker MN, Diamond G, Verghese MW, et al. CD14-dependent lipopolysaccharide-induced beta-defensin-2 expression in human tracheobronchial epithelium. J Biol Chem 2000; 275: 29731-36.

13. Sörensen $0 \varepsilon$, Thapa DR, Rosenthal A, et al. Differential regulation of $\beta$-defensin expression in human skin by microbial stimuli. J Immunol $2005 ; 174: 4870-9$. 
14. Liu L, Roberts AA, Ganz T. By IL-1 signaling, monocyte-derived cells dramatically enhance the epidermal antimicrobial response to lipopolysaccharide. J Immunol 2003 ; 170 : 575-80.

15. Krisanaprakornkit $S$, Kimball JR, Weinberg $A$, et al. Inducible expression of human beta-defensin 2 by Fusobacterium nucleatum in oral epithelial cells: multiple signaling pathways and role of commensal bacteria in innate immunity and the epithelial barrier. Infect Immun 2000 ; 68 : 2907-15.

16. Chung WO, Dale BA. Innate immune response of oral and foreskin keratinocytes: utilization of different signaling pathways by various bacterial species. Infect Immun $2004 ; 72$ : 352-8.

17. Harder J, Bartels J, Christophers $\varepsilon$, et al. Isolation and characterization of human beta-defensin-3, a novel human inducible peptide antibiotic. J Biol Chem $2001 ; 276$ : 5707-13.

18. Garcia JR, Jaumann F, Schulz S, et al. Identification of a novel, multifunctional beta-defensin (human beta-defensin 3) with specific antimicrobial activity. Its interaction with plasma membranes of Xenopus oocytes and the induction of macrophage chemoattraction. Cell Tissue Res 2001 ; 306 : 257-64

19. Agerberth B, Gunne H, Odeberg J, et al. FALL-39, a putative human peptide antibiotic, is cysteine-free and expressed in bone marrow and testis. Proc Natl Acad Sci USA 1995 ; 92 : 195-9.

20. Zanetti $M$. The role of cathelicidins in the innate host defenses of mammals. Curr Issues Mol Biol $2005 ; 7:$ 179-96.

21. Frohm M, Agerberth B, Ahangari G, et al. The expression of the gene coding for the antibacterial peptide LL-37 is induced in human keratinocytes during inflammatory disorders. J Biol Chem 1997 ; $272: 15258-63$

22. Murakami $M$, Ohtake $T$, Dorschner RA, et al Cathelicidin anti-microbial peptide expression in sweat, an innate defense system for the skin. J Invest Dermatol 2002 ; 119 : 1090-5

23. Gombart AF, Borregaard N, Koeffler HP. Human cathelicidin antimicrobial peptide (CAMP) gene is a direct target of the vitamin $D$ receptor and is strongly up-regulated in myeloid cells by $1,25-$ dihydroxyvitamin D3. FASEB J 2005 ; 19 : 1067-77.

24. Nizet $V$, Ohtake $T$, Lauth $X$, et al. Innate antimicrobial peptide protects the skin from invasive bacterial infection. Nature $2001 ; 414: 454-7$.

25. Henseler T, Christophers $\varepsilon$. Disease concomitance in psoriasis. J Am Acad Dermatol 1995 ; 32 : 982-6

26. Nomura I, Goleva $\varepsilon$, Howell MD, et al. Cytokine milieu of atopic dermatitis, as compared to psoriasis, skin prevents induction of innate immune response genes. J Immunol 2003; 171:3262-9.

27. Ong Py, Ohtake T, Brandt C, et al. Endogenous antimicrobial peptides and skin infections in atopic dermatitis. N Engl J Med 2002 ; 347 : 1151-60.

28. Giesbrecht P, Kersten T, Maidhof H, Wecke J. Staphylococcal cell wall: morphogenesis and fata variations in the presence of penicillin. Microbiol Mol Biol Rev $1998 ; 62$ : 1371-1414.

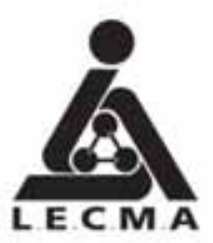

Ligue

Européenne

Contre la

Maladie d'Alzheimer

\section{APPEL AUX BOURSES POUR LA RECHERCHE BIOMÉdiCALE Et CLINIQUE FONDAMENTALE SUR LA MALADIE D'ALZHEIMER}

La Ligue Européenne Contre la Maladie d'Alzheimer (LECMA) octroie des bourses pour la recherche scientifique dans le domaine de la maladie d'Alzheimer. Sont acceptées les demandes de scientifiques d'institutions françaises.

Les approches innovantes et révolutionnaires sont encouragées. Les candidats doivent avoir une expérience dans le domaine de la recherche sur la maladie d'Alzheimer, Les jeunes chercheurs doivent être associés à un laboratoire expérimenté en matière de recherche dans le domaine de la maladie d'Alzheimer. Les chercheurs d'un même laboratoire peuvent faire leur demande pour autant que les projets de recherche proposés ne se chevauchent pas.

Les demandes de bourse standard sont acceptées jusqu'à concurrence de $\mathbf{\epsilon} 80000$ pour deux ans avec un maximum de $€ 40000$ pour un an.

Les demandes de bourse pour des projets pilotes par de jeunes chercheurs sont acceptées jusqu'à concurrence de $\boldsymbol{\epsilon} \mathbf{4 0 0 0 0}$ pour deux ans avec un maximum de $€ 20000$ pour un an. Sont éligibles dans cette catégorie les jeunes chercheurs avec un minimum de 2 ans et un maximum de 6 ans d'expérience après la réussite de leur thèse.

Lessubventions peuvent êtreutilisées pour couvrir les frais de personnel, d'équipement et de fournitures. Elles seront mises a disposition en novembre 2006.

Les subventions sont octroyées sur base du mérite scientifique du projet de recherche proposé et de son utilité pour améliorer notre compréhension de la maladie d'Alzheimer. Les demandes seront examinées par le comité consultatif scientifique européen, composés de pairs placés sous la présidence du Dr Frédéric Checler.

Les demandes doivent être rentrées par e-mail (sous forme de fichier Word) à e.wiese alzheimer-forschung.de et 5 exemplaires par la poste, (un original et quatre copies certifiées conformes), au plus tard le 10 avril 2006, date de la poste faisant foi, à l'adresse suivante:

\section{Ligue Européenne Contre la Maladie d'Alzheimer 21, PLACE de La RépubLQue, 75003 Paris}

Les formulaires de candidatures et des informations détaillées seront disponibles en janvier 2006 le site Internet: www.MaladieAlzheimer.fr

Pour toute question, veuillez prendre contact avec Dr Ellen Wiese, Research Grant Manager au numéro +49-211-86206621 ou par e-mail: e.wiese@alzheimerforschung.de.

En 2005, la LECMA a attribué des subventions à trois projets à concurrence d'un montant de $€ 240000$.

\section{TIRÉS À PART}

J.M. Schröder

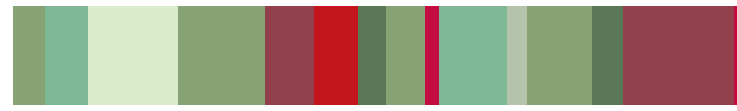

